\title{
¿Qué aprendimos del terremoto de abril de 2016? Respuesta del Gobierno ecuatoriano a la emergencia
}

\author{
What Did We Learn from April 2016 Earthquake? \\ Ecuadorian Government Response to the Emergency \\ ¿O que aprendemos com o terremoto de abril de 2016? \\ Resposta do governo equatoriano frente à emergência
}

\section{Cristian Castillo Peñaherrera}

Universidad del Azuay. Cuenca, Ecuador ccastillo@uazuay.edu.ec

https://orcid.org/0000-0003-0038-1483

\author{
Juan Santillán Berrones \\ Universidad Complutense de Madrid. Madrid, España \\ jsantill@ucm.es \\ https://orcid.org/0000-0002-9977-1096
}

DOI: https://doi.org/10.32719/25506641.2021.10.2

Recibido: 5 de agosto de 2020 - Revisado: 13 de noviembre de 2020

Aceptado: 25 de enero de 2021 • Publicado: 1 de julio de 2021

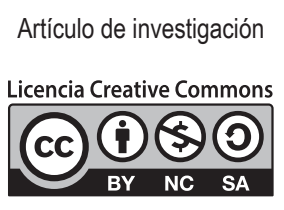




\section{Resumen}

Este artículo analiza la capacidad de respuesta del gobierno del Ecuador ante la emergencia producida por el terremoto del 16 de abril de 2016. A partir de una investigación de carácter cualitativo explicativo, se estudia el desempeño de las organizaciones públicas del gobierno central para atender emergencias de alta complejidad. Dado que el país se encuentra en una zona tectónica complicada, la posibilidad de ocurrencia de hechos como el que motiva este estudio es alta. Es deber de todos los niveles de gobierno desarrollar estrategias para enfrentar situaciones de emergencia. De acuerdo con la literatura, la prevención y la preparación anticipada del Ejecutivo y otros actores es la mejor herramienta para hacer frente a los desafíos que generan dichas situaciones. Para ello, es necesario desarrollar las capacidades organizacionales de respuesta de todos los sectores de política pública que deben participar activamente en la gestión de una emergencia. El estudio identifica focos de mejora en el diseño organizacional, de asignación de roles y responsabilidades de las diferentes organizaciones públicas involucradas.

Palabras clave: administración pública, gobierno central, institucionalización, prevención de desastres, sismos.

JEL: H1 Estructura y ámbito del sector público.

\section{Abtract}

This article analyzes the response capacity of the Ecuadorian government facing the emergency produced by the earthquake on April 16, 2016. Based on a qualitative and explanatory research, the performance of public organizations from the central government to attend emergency situations is studied. High complexity. Given that the country is located in a complicated tectonic zone, the possibility of occurrence of events such as the one that motivates this study is high. It is the duty of all levels of the government to develop strategies to face emergency situations. According to the literature, the prevention and advance preparation of the Executive and other actors is the best tool to face these situation challenges. For this, it is necessary to develop the organizational response capacities of all the public policy sectors that must actively participate in an emergency management, which includes a wide range of government action sectors. The study identifies sources of improvement in the organizational design, assignment of roles and responsibilities of the different public organizations involved; Improvements are also suggested in the design of protocols and procedures, as well as in the prevention and respo

Keywords: public administration, central government, institutionalization, disaster prevention, earthquakes.

JEL: H1 Structure and scope of government. 


\section{Resumo}

Este artigo analisa a capacidade de resposta do governo do Equador frente à emergência produzida pelo terremoto de 16 de abril de 2016. A partir de uma pesquisa de caráter qualitativo-explicativo, estuda-se o desempenho das organizações públicas do governo central para atender emergências de alta complexidade. Dado que o país se encontra em uma zona técnica complicada, a possibilidade de ocorrência de fatos como o que motiva este estudo é alta. É dever de todos os níveis do governo desenvolver estratégias para enfrentar situações de emergência. De acordo com a literatura, a previsão e preparação antecipada do Executivo e de outros atores é a melhor ferramenta para enfrentar os desafios que gerados por tais situações. Para tanto, é necessário desenvolver as capacidades organizacionais de resposta de todos os setores de política pública que devem participar ativamente na gestão de uma emergência, o que inclui um amplo leque de setores de ação governamental. $\mathrm{O}$ estudo identifica focos de melhoria no desenho organizacional, de designação de papéis e responsabilidades das diferentes organizações públicas envolvidas, além de sugerir melhorias no desenho de protocolos e procedimentos, assim como nos mecanismos de prevenção e resposta frente a situações de emergência ou desastre.

Palavras-chave: administração pública, governo central, institucionalização, prevenção de desastres, sismos.

JEL: H1 Estrutura e abrangência do setor público.

\section{Introducción}

las $18 \mathrm{~h} 58$ del 16 de abril de 2016, Ecuador sufrió un terremoto de
magnitud $7,8 \mathrm{Mw}$ en la escala Richter a $20 \mathrm{~km}$ de profundidad. El
movimiento telúrico se prolongó por 75 segundos. Durante los días posteriores al hecho, se registraron seis réplicas de magnitud de entre 6,0 y 6,76 grados (EC SENPLADES 2016). Como resultado, fallecieron 661 personas, 6274 resultaron heridas (EC Secretaría de Gestión de Riesgos 2016) y un total de 430.000 fueron afectadas (350.000 requirieron ayuda humanitaria y 80.000 fueron desplazadas por la pérdida o daño de sus hogares) (ACNUR 2017), así como la devastación total o parcial de centros poblados como Jama o Pedernales. La emergencia generó problemas de seguridad, salud, falta de acceso a alimentos o servicios básicos en amplios sectores de la población de las provincias de Esmeraldas, Manabí, Santo Domingo de los Tsáchilas, Guayas, Los Ríos y Santa Elena, que debieron ser atendidos por las organizaciones del Estado local, provincial y nacional. 
El Ecuador está ubicado en el punto de encuentro de las placas de Nazca y Sudamérica, una de las zonas tectónicas más complejas del mundo. La ocurrencia de fenómenos naturales es usual en el país. Entre 1982 y 2016 se dieron ocho eventos que la literatura reconoce como catastróficos — por lo inesperado y de gran afectación-(Kapucu 2009). Estos eventos fueron inundaciones, erupciones volcánicas y terremotos que perjudicaron, en el menor de los casos, a 128.000 personas (erupción del volcán Tungurahua de 2002), y en el peor, a más de 700.000 (El Niño de 1982) (ACNUR 2017). En todos los eventos, la población pobre de las áreas rurales y pequeños poblados resultó la más afectada (FAO 2008). Ante la constante de eventos naturales complejos, el Estado debería contar con un sistema de gestión integral de emergencias que contenga las etapas básicas de preparación social; gestión de la respuesta organizacional, recursos e inventarios; respuesta inmediata, evaluación situacional, comando y control, y recuperación y respuesta poscrisis (Wallace y De Balogh 1985). Este estudio aporta con insumos para la mejora de este sistema.

El artículo analiza, en particular, la respuesta del gobierno nacional del Ecuador ante la emergencia; observa cuáles son los ámbitos en los que la respuesta dio resultados y en cuál la respuesta no generó resultados o fueron insuficientes. La investigación incluyó entrevistas a interlocutores que representaban al gobierno central, gobiernos locales, sociedad civil y organismos de emergencia. Además, se incluyó un análisis de fuentes secundarias para comprender la forma en la que otros países que han experimentado eventos similares, reaccionaron.

Se parte del supuesto de que la respuesta del gobierno central fue eficaz debido a los esfuerzos de consolidación de sus organizaciones públicas, en el marco de la política de fortalecimiento institucional llevada a cabo por el gobierno en el período 2007-2017.

\section{Antecedentes y revisión teórico-conceptual}

Los Estados requieren de una estrategia integral para responder a las condiciones cambiantes del entorno (Banco Mundial 1997). Esta estrategia requiere capacidad para acomodar sus facultades actuales y aumentar aquellas necesarias para enfrentar los problemas públicos, lo cual involucra la 
revitalización de las instituciones públicas. La gestión que la administración realiza sobre las líneas de acción gubernamental, ya sean de planificación o integración de escenarios de gestión en una estrategia unificada y alineada, puede facilitar el alineamiento estratégico gubernamental y eliminar la dispersión sobre las actividades que se deben desarrollar para la atención de desastres y emergencias (Báez Mancera 2013).

El gobierno ecuatoriano fue efectivo en crear un conjunto de nuevas instituciones estatales, aunque con un impacto práctico heterogéneo (Bowen 2020). En particular, se identificaron dos esfuerzos relevantes por fortalecer el sistema de gestión de emergencias, a través de la creación de dos organizaciones públicas complementarias entre ellas, contrario a lo sucedido en otras experiencias, en las que la gestión de emergencias no formó parte del encuadre principal del desarrollo institucional (Petak 1985).

Por un lado, el Servicio Integrado de Seguridad ECU 911 (ECU 911) creado por Decreto Ejecutivo No. 988 en diciembre de 2011 (EC 2011) como espacio de coordinación de las organizaciones públicas que operan diferentes procesos de la gestión de emergencias: salud pública, bomberos, Policía Nacional, gestión de tránsito, entre otros; y por otro, la Secretaría Nacional de Gestión de Riesgos que fue creada por Decreto Ejecutivo No. 42 en septiembre de 2009 (EC 2009) como órgano de decisión política para la disminución de las vulnerabilidades de los territorios ante diferentes tipos de riesgos naturales o antrópicos, ayudaron a trascender la mirada del sistema, más allá de las instituciones tradicionales como son la policía o el departamento de bomberos (Eburn 2017), así como con un enfoque proactivo de la acción pues, la forma correcta de gestión es la mitigación de los riesgos y el mejoramiento de las capacidades de respuesta.

La capacidad institucional para atender emergencias que pueda desarrollar la administración pública, se puede explicar, entre otras razones, por la prioridad política que se le asigne a su gestión, la capacidad de negociación de los responsables de la política pública, las condiciones económicas y sociales presentes y las capacidades específicas de cada nivel de gobierno (Ok Choi 2004). En este caso, se infiere que la mejora institucional para la atención de emergencias fue importante. En la figura 1 se aprecia un incremento del 2,01\% en los presupuestos entre los períodos 2008-2012 y 2013-2017. 
Figura 1

Evolución de los egresos permanentes por entidad (en millones de dólares)

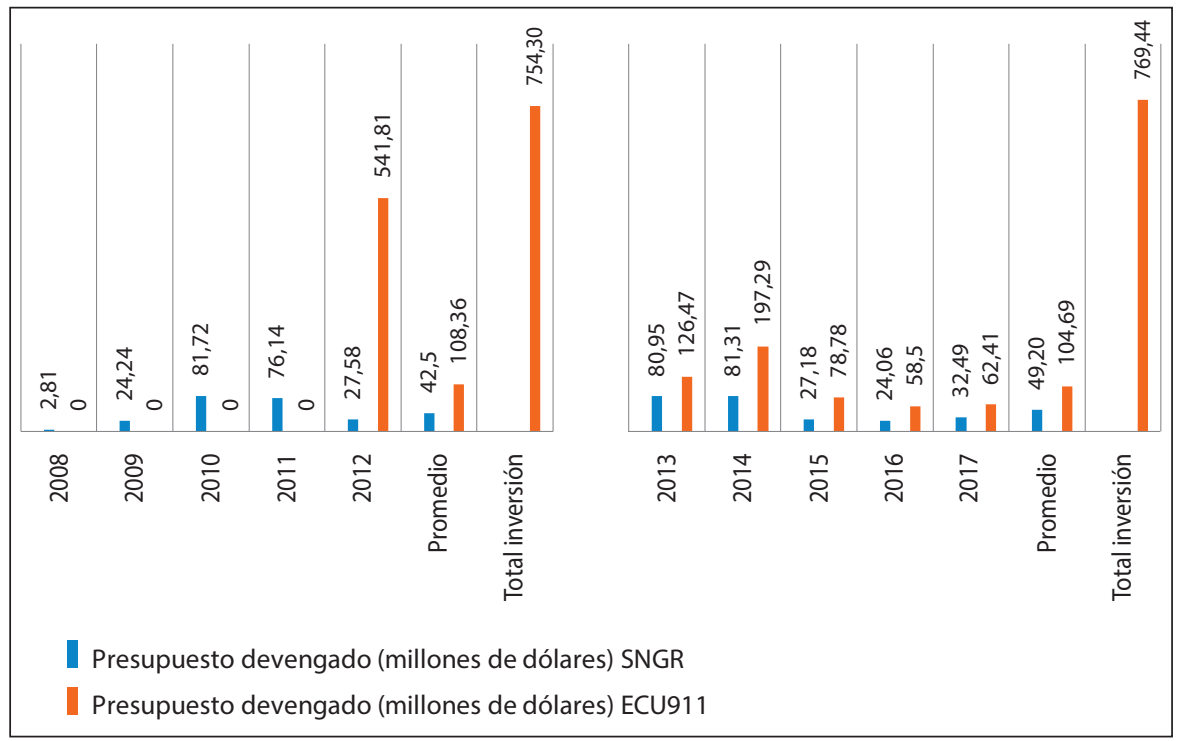

Fuente: adaptado de Ministerio de Economía y Finanzas (2017).

La mejora de la capacidad de respuesta se explica también por la forma en la que se han fortalecido otros sectores de gestión gubernamental vinculados con distintas fortalezas requeridas para atender los eventos catastróficos. Por ejemplo, la creación del Ministerio de Electricidad y Energías Renovables o los esfuerzos de fortalecimiento organizacional del sistema de empresas públicas de generación y distribución eléctrica impulsados desde 2008 (Pazmiño 2020), hicieron posible que los servicios públicos, provistos por estas empresas, tuvieran desempeños que permitieron a amplios sectores afectados por el evento, que puedan recuperar el servicio en poco tiempo.

Como cualquier ámbito de la administración pública donde existe más de una organización o más de un nivel de gobierno involucrado en su gestión, en el sector de la atención de emergencias es visible la importancia de los mecanismos de coordinación entre organizaciones, entendidas como formas de cooperación mutua que se articulan para atender desastres modernos 
complejos y con gran potencial de impacto adverso (McEntire et al. 2002; Henderson y Charbonneau 2016; Cadillo 2019). La atención de estos eventos requiere autoridad compartida y procesos colaborativos (Waugh y Streib 2006) que trascienden las formas tradicionales de gestión de otros sectores de la cosa pública ajenos a la gestión de riesgos.

Los modelos actuales de política pública constituyen un sistema que incluye comunidades resistentes, resilientes y sostenibles. En el ámbito de la gestión de riesgos, las políticas públicas deben contemplar acciones preventivas y con un enfoque multidisciplinar (Beydoun et al. 2018; Nespeca et al. 2020; Waugh y Streib 2006), puesto que la preparación insuficiente y la falta de articulación puede incrementar la vulnerabilidad de las comunidades (Weichselgartner 2001).

Se evidencia un incremento de los esfuerzos en las ciencias sociales por analizar la problemática de la gestión de emergencias, enfocándose en el análisis de la vulnerabilidad como elemento determinante en la causa de los desastres o la configuración de riesgos (Kapucu 2011; Lavell y Lavell 2020). El aporte de este enfoque fue la incorporación de la sociedad como elemento activo y responsable en la formación de riesgos y su mitigación, a través de procesos económicos, sociales, políticos o ambientales (Gellert-de Pinto 2012) que incrementan o disminuyen el riesgo ante un desastre.

En los procesos actuales de planificación para construir entornos urbanos sostenibles se debe incorporar el análisis preventivo de las amenazas naturales y las variables de espacio y tiempo de los desastres con relación a las situaciones ex ante, durante y ex post, y las acciones que se desprenden de cada una, requiere de enfoques integrados o interdisciplinares (Ferrando 2003).

Finalmente, la gestión de emergencias es un sector de política pública altamente profesionalizado que incluye el desarrollo de capacidades como el pronóstico de inundaciones, evacuación, gestión de incidentes, logística, refugios o la gestión de recursos antes del suceso (Weichselgartner 2001), búsqueda y rescate, servicios médicos o recuperación de la cotidianidad inmediatamente después del suceso (Waugh y Streib 2006). El sector debe incluir, además: mitigación de riesgos identificados, preparación de la sociedad ante desastres, actividades de búsqueda y rescate, y recuperación o restauración de servicios básicos. 


\section{Metodología}

Esta investigación se sustenta en el método de estudio de caso con enfoque cualitativo explicativo (Castro Monge 2010). Recupera información de entrevistas a interlocutores válidos y de fuentes oficiales con el objetivo de establecer las razones que motivaron el comportamiento de las organizaciones del gobierno nacional que intervinieron en la gestión de respuesta ante la emergencia; así como también identificar lecciones aprendidas que puedan mejorar el comportamiento futuro de las organizaciones públicas en casos similares. Para obtener la información, se utilizaron las siguientes técnicas:

1. Revisión en fuentes oficiales como reportes de resultados de la gestión gubernamental de respuesta ante otros eventos (Terremotos de Perú, 2007, Chile, 2010, y el terremoto y tsunami de Japón, 2011) así como los manuales y protocolos de atención a emergencia de la Oficina de Coordinación de Asuntos Humanitarios de las Naciones Unidas (OCHA, por sus siglas en inglés); Guías 2015 del Grupo Asesor Internacional de Operaciones de Búsqueda y Rescate (INSARAG, por sus siglas en inglés). Además, se revisaron los protocolos de atención de emergencia del Banco Mundial y la Cruz Roja.

2. Entrevistas a doce autoridades y funcionarios del gobierno del período de estudio que, por sus funciones lideraron las labores de respuesta; seis autoridades de gobiernos locales de las zonas afectadas por el terremoto que lideraron en sus territorios las labores de respuesta; cinco líderes de organismos de rescate y emergencia que acudieron a realizar labores de rescate, inmediatamente sucedido el evento; y cuatro miembros de la sociedad civil que, de manera voluntaria, organizaron labores de rescate y aprovisionamiento de los territorios que tuvieron mayores problemas.

La selección de los entrevistados se realizó con base en la posibilidad de contacto e interés y deseo de compartir sus percepciones sobre el fenómeno estudiado. El instrumento utilizado para todas las entrevistas fue una guía semiestructurada de temas con variaciones en función del interlocutor. La guía permitió entrevistas de hasta 60 minutos. La información recopilada se procesó en una matriz por eje temático hasta obtener saturación teórica (Lúquez de Camacho 2016), lo que permitió incrementar la fiabilidad de cada criterio identificado. 
El análisis de la información se realizó a través de una línea temporal ${ }^{1}$ desde el minuto uno hasta la hora $120^{2}$ después del suceso, que sirvió para identificar hechos concretos a partir de los cuales se pudo identificar comportamientos específicos de las organizaciones intervinientes.

\section{Resultados}

\section{Otros eventos en la región}

Para mejorar la comprensión de la situación estudiada, la investigación levantó información de otros sismos acontecidos en Sudamérica y los tiempos que les tomó recuperar servicios básicos como energía o agua potable, que se constituyen en sectores altamente vulnerables ante la ocurrencia de eventos como el que se investiga.

De esta revisión se encontró que la recuperación de servicios básicos como en el caso de la energía, tomó, tanto en el caso estudiado como en el del terremoto de Chile de 2010, un lapso de diez días hasta recuperar el servicio en más del $90 \%$ de las áreas urbanas afectadas (Rojas Hoppe y Díez Lorente 2013; OPS/OMS 2010). En el caso de la provisión de agua potable, a los organismos estatales ecuatorianos encargados les tomó diez días recuperar la provisión total de servicio (PAHO 2016); y en el caso del terremoto del Perú, en ese mismo lapso, la recuperación del servicio de agua potable abarcó el $80,1 \%$ de las áreas afectadas (PAHO 2007).

\section{Normas internacionales para atención de emergencias ocasionadas por terremotos}

La ONU, a través de la OCHA y de INSARAG, han elaborado guías que sirven de referencia para quienes tienen la responsabilidad de tomar decisio-

1. Línea temporal se encuentra en el anexo: https://doi.org/10.6084/m9.figshare.13669034.v1.

2. A partir del quinto día, las autoridades consideraron superada la fase de emergencia y empezaron las tareas de reconstrucción. 
nes políticas y administrativas, y que colaboran en el aseguramiento de asistencia de calidad en las actividades de búsqueda y rescate para salvar vidas de forma inmediata a la ocurrencia de un desastre. Las normas se componen de tres volúmenes: i) Política de las Guías de INSARAG; ii) Preparación y respuesta; y, iii) Guía de operaciones en el terreno (OCHA 2015).

Aunque se reconoce que la calidad de la asistencia ante situaciones de emergencia mejora cuando se fortalecen las capacidades organizativas de los actores locales (Sanderson y Ramalingam 2015); el fenómeno dejó en evidencia que, aunque varios de los cuerpos de rescate del Ecuador contaban con autonomía y entrenamiento, las tareas de rescate se vieron limitadas por un desconocimiento general de las guías INSARAG y de las guías del Centro de Coordinación de Operaciones en el sitio (OSOCC, por sus siglas en inglés), salvo excepciones identificadas como en el caso de los Cuerpos de Bomberos de Quito, Guayaquil y Cuenca.

Otro problema identificado fue que los requerimientos de equipos internacionales de Búsqueda y Rescate Urbano (USAR, por sus siglas en inglés) no respondieron a necesidades reales. La poca coordinación institucional entre la SGR y otras entidades gubernamentales dificultó la efectiva recepción y coordinación de equipos de profesionales (SGR e INSARAG 2016). Las autoridades nacionales y algunos de los equipos internacionales que asistieron la emergencia poseían limitado conocimiento del uso del OSOCC virtual ${ }^{3}$ lo que redujo la capacidad de identificación y requerimiento de equipos USAR internacionales en estado de espera para asistir al evento. Esto ocasionó que los equipos que arribaron tuvieran capacidades operativas heterogéneas, lo que, a su vez, provocó formas desorganizadas de trabajo, áreas con sobrecarga de ayuda logística, en comparación con otras y ayuda que no llegó a otras zonas.

\section{Análisis de la información recopilada}

Los problemas se analizaron de acuerdo con estas variables: diseño organizacional, protocolos y procedimientos, y prevención y respuesta.

3. https://vosocc.unocha.org: plataforma electrónica para la coordinación internacional de equipos de respuesta. 
El diseño organizacional es entendido como la estructura y las formas de coordinación (Rodríguez 2002; Ramio 1999) que se pueden identificar para atender emergencias de gran envergadura. Esto permite identificar el potencial de eficacia de la respuesta, pues está limitada por la forma en la que se dan los mecanismos de coordinación (Mintzberg 2003) establecidos en el sistema. En este caso, las organizaciones que conforman el Comité de Operaciones de Emergencia (COE) en el nivel nacional son casi en su totalidad, miembros del gobierno central. Este mecanismo de coordinación varía según el nivel territorial, como se explica en la tabla 1.

Tabla 1

\section{Conformación de plenarios del COE de acuerdo con el nivel activado}

\begin{tabular}{|c|c|}
\hline Plenario del CGR / COE Nacional & Plenario del CGR / COE Cantonal \\
\hline Preside el presidente de la República & - Preside el Alcalde. \\
\hline Ministros Coordinadores & - Jefe político. \\
\hline $\begin{array}{l}\text { Ministros sectoriales, agencias nacionales } \\
\text { de tránsito, control minero y control de hidrocarburos }\end{array}$ & $\begin{array}{l}\text { - Representantes de ministerios presentes } \\
\text { en el cantón. } \\
\text { - Representante de la Unidad de Gestión } \\
\text { de Riesgos municipal. } \\
\text { - Empresas municipales. }\end{array}$ \\
\hline Secretaría de Gestión de Riesgos & $\begin{array}{l}\text { - Delegados de la SGR. } \\
\text { - Jefes de entidades de socorro (Bomberos, } \\
\text { Comisión de Tránsito, Cruz Roja). }\end{array}$ \\
\hline Secretaría Técnica de Discapacidades (SETEDIS) & - Delegados de la SETEDIS. \\
\hline Ministro de Defensa Nacional (MIDENA) & $\begin{array}{l}\text { - Delegado de las Fuerzas Armadas (FF. } \\
\text { AA.) en el cantón. }\end{array}$ \\
\hline Ministro del Interior & $\begin{array}{l}\text { - Delegado de la Policía Nacional } \\
\text { en el cantón. }\end{array}$ \\
\hline $\begin{array}{l}\text { Presidente de Asociación de Municipalidades } \\
\text { del Ecuador (AME) }\end{array}$ & $\begin{array}{l}\text { - Representante cantonal de las Juntas } \\
\text { Parroquiales. }\end{array}$ \\
\hline Otros a criterio del ente rector & $\begin{array}{l}\text { - Coordinadores de las mesas técnicas } \\
\text { - Otros a criterio del Comité de Gestión } \\
\text { de Riesgos / COE Cantonal. }\end{array}$ \\
\hline
\end{tabular}

Fuente: SGR (2014). 
Cuando, por la dimensión de una emergencia, se decreta un estado de excepción que corresponde al nivel máximo de alerta por emergencia, el COE se convoca por disposición de la máxima autoridad de la SGR. En este caso, sucedió que se activaron simultáneamente el Comité Nacional, el Comité provincial de Manabí y el Comité cantonal de Manta; sin que existieran enlaces entre cada nivel. Esto devino en actuaciones paralelas, superposición de acciones y toma de decisiones no coordinadas (Patiño 2016, entrevista personal).

Si bien la conformación del mecanismo de coordinación en el Manual de la SGR es taxativa, las formas de coordinación no necesariamente lo son; instituciones como las Fuerzas Armadas, el Ministerio de Inclusión Económica y Social (MIES), la SGR, la Secretaría Nacional del Agua (SENAGUA), o la Dirección de Movilización del Comando Conjunto de las Fuerzas Armadas (DIRMOV) no coordinaron sus actuaciones con la rapidez requerida. Por ejemplo, aunque se disponía la conformación de mesas técnicas de coordinación, entraron en operación con retrasos de hasta cuatro días (Navas 2016, entrevista personal); otros ejemplos son la administración del voluntariado que arribó al lugar del desastre con o sin invitación, el manejo de la cooperación internacional o la remoción de escombros (Patiño 2016, entrevista personal).

Finalmente, es importante señalar que la literatura reconoce la relevancia que tienen los niveles locales y comunitarios en la respuesta inmediata (Schneider 2008; Jung, Song y Jun Park 2018). No obstante, en el caso analizado se encontró que, ante la magnitud del desastre, el presidente de la República dispuso que los ministros del gobierno nacional actúen como últimos responsables en las ciudades en donde se había concentrado el mayor impacto del evento, por sobre las propias capacidades de los líderes locales. Esto generó ruido entre los organismos proveedores de servicios y requirentes porque el acceso a aprovisionamiento, en ocasiones, no se hizo con base en información, sino sobre presión de las autoridades. Se puede inferir que la magnitud del evento puso en evidencia vacíos en el Manual de la SGR, lo que llevó a algunas autoridades a inobservar, en ocasiones, los procedimientos establecidos.

La segunda variable de análisis es la de protocolos y procedimientos. En algunos casos, estos eran confusos; en otros, no existían al momento de 
terremoto. Por ejemplo, se identificó que no existían manuales de procedimiento que indiquen las tareas de comunicación a cumplir, ni quiénes debían ejecutarlas.

El Manual de la SGR disponía, de manera general, que el presidente del COE notifique a la oficina de la Secretaría Nacional de Comunicación (SECOM) en la provincia para que un funcionario apoye el relacionamiento con los medios (SGR 2014), no obstante, la SECOM no tenía manuales ante situaciones de emergencia. De acuerdo con los entrevistados, esto provocó retrasos cuando el vicepresidente de la República realizó las primeras comparecencias ante la prensa (Glas 2016, entrevista personal). A ese momento, la SECOM no contaba con guiones de referencia para orientar la emisión (Barriga 2016, entrevista personal), ni con una guía de acciones previas a realizar que dispusiera cómo debían enlazarse los medios de comunicación o establecer vocerías previamente entrenadas. Es importante notar que la gestión de crisis puede mejorar cuando la ciudadanía tiene acceso oportuno a información confiable que permita conocer la dimensión del evento, la posibilidad de réplicas u otros eventos esperados (Moorthy, Benny y Gill 2018; UNESCO 2011) lo cual disminuye la ansiedad y la circulación de información errónea.

La experiencia puso en evidencia que el Manual de la SGR no tenía respuestas estructuradas a eventos de gran envergadura como un terremoto. Al respecto solo contenía indicaciones para los procesos de adquisiciones en situación de emergencia establecidos en la Ley de Contratación Pública vigente a la fecha del suceso y no hacía referencia a los roles y responsabilidades de los operadores para atender las diferentes aristas de los problemas que se generan por este tipo de desastres. Otros problemas identificados sobre protocolos y procedimientos se indican en la tabla 2.

La última variable analizada fue la de prevención y respuesta en la cual se constató varias carencias de gestión que eran responsabilidad de la SGR. Por ejemplo, no existían procedimientos generales para atender las primeras 6 a 72 horas; no se realizaron simulacros para probar la capacidad de respuesta gubernamental, ni el número suficiente de simulaciones teóricas; y finalmente, varios directivos públicos no tuvieron entrenamiento suficiente o recursos para responder ante una situación como la descrita en este caso (Dueñas 2016, entrevista personal). 
Tabla 2

Análisis de responsabilidades, protocolos, procedimientos por institución y problemas identificados

\begin{tabular}{|c|c|c|}
\hline Ministerio & $\begin{array}{l}\text { Responsabilidad / } \\
\text { procedimiento según } \\
\text { Manual de SGR }\end{array}$ & Problema identificado \\
\hline $\begin{array}{l}\text { Gestión } \\
\text { de Riesgos }\end{array}$ & $\begin{array}{l}\text { - Declara estados de alerta. } \\
\text { - Evalúa y da seguimiento } \\
\text { a decisiones del COE. } \\
\text { - Coordina llegada de ayuda. } \\
\text { - Actividades de apoyo a la Dirección } \\
\text { de Monitoreo de la SGR a los COE } \\
\text { provinciales. }\end{array}$ & $\begin{array}{l}\text { - Ausencia de protocolos y procedimien- } \\
\text { tos. } \\
\text { - Falta de simulacros a nivel nacional } \\
\text { para testeo de respuesta gubernamen- } \\
\text { tal. } \\
\text { - No asume coordinación de inventario y } \\
\text { distribución de ayuda internacional. }\end{array}$ \\
\hline Comunicación & $\begin{array}{l}\text { - Acompaña y asesora al delegado } \\
\text { del COE y SGR para informar a la } \\
\text { población del estado de la situación } \\
\text { de emergencia. }\end{array}$ & $\begin{array}{l}\text { - Ausencia de procedimientos y formatos } \\
\text { de comunicación (líneas argumentales). } \\
\text { - Activación de equipos técnicos depen- } \\
\text { den de decisión de actores. }\end{array}$ \\
\hline $\begin{array}{l}\text { Relaciones } \\
\text { Exteriores }\end{array}$ & $\begin{array}{l}\text { - Solicita y coordina con la SGR } \\
\text { la ayuda internacional necesaria. }\end{array}$ & $\begin{array}{l}\text { - Falta de procedimientos para la gestión } \\
\text { de recepción, consolidación, envío } \\
\text { de donaciones, tanto oficiales } \\
\text { como voluntarias. } \\
\text { - Asistencia humanitaria de la SGR. }\end{array}$ \\
\hline Vivienda & $\begin{array}{l}\text { - Evalúa daños y análisis } \\
\text { de las necesidades de vivienda } \\
\text { e infraestructura sanitaria. }\end{array}$ & $\begin{array}{l}\text { - Ausencia de protocolos de gestión } \\
\text { de infraestructura privada. } \\
\text { - Metodología de semaforización se } \\
\text { aplicó sobre la marcha (uso, arreglo, } \\
\text { demolición de infraestructuras). }\end{array}$ \\
\hline Defensa & $\begin{array}{l}\text { - Apoya en logística y seguridad. } \\
\text { - Apoya acciones de seguridad } \\
\text { e integridad de la población. } \\
\end{array}$ & $\begin{array}{l}\text { - No actuaron con celeridad, ni se ajus- } \\
\text { taron a los procedimientos establecidos } \\
\text { en el Manual de la SGR. }\end{array}$ \\
\hline Justicia & $\begin{array}{l}\text { - Informa y orienta a la población } \\
\text { sobre sus derechos y vela } \\
\text { por su cumplimiento } \\
\text { en situaciones de albergue } \\
\text { o alojamiento temporal. }\end{array}$ & $\begin{array}{l}\text { - Ausencia de procedimientos específicos } \\
\text { para casos de emergencia en centros } \\
\text { de rehabilitación social. }\end{array}$ \\
\hline $\begin{array}{l}\text { Fiscalía } \\
\text { General } \\
\text { del Estado }\end{array}$ & - No consta en el Manual de la SGR. & $\begin{array}{l}\text { - No tiene procedimientos definidos para } \\
\text { levantamiento de cadáveres en situacio- } \\
\text { nes de emergencias. }\end{array}$ \\
\hline
\end{tabular}

Elaboración propia. 


\section{Mejoras al sistema de atención a emergencias}

El diseño organizacional del sistema podría mejorarse. La evidencia sugiere que resulta ineficaz manejar los asuntos de la gestión de riesgos como un organismo de carácter político (vale recordar que la Secretaría tenía rango de ministerio). Estos asuntos parecen estar muy vinculados con los de la política de seguridad interior, y podrían ser mejor instrumentados a través de organismos técnicos especializados. Varios entrevistados notaban la confusión que generaba la existencia de la SGR y del Sistema Integrado de Seguridad ECU-911 (Maldonado 2016, entrevista personal) y sugerían un mejor resultado si ambos organismos hubieran estado unificados.

Los datos obtenidos también apuntan hacia la necesidad de incorporar un enfoque de gobernanza efectiva en la estructuración del COE en todos sus niveles. La capacidad de atender emergencias como un terremoto mejora cuando participan actores locales y de otros sectores de la sociedad que alimentan la información y mejoran la calidad de las decisiones (Levi-Faur 2012; Lein et al. 2009). En este sentido, es muy importante que en este espacio de coordinación puedan participar actores sociales que aporten información técnica y territorializada (Glas 2016, entrevista personal).

Existe una relación estrecha entre la calidad de la respuesta ante la emergencia y las capacidades locales creadas con anterioridad (McEntire et al. 2002). La evidencia sugiere que no todas las localidades tenían fortalecidas estas capacidades. Los cuerpos de bomberos, por ejemplo, presentaron asimetrías muy marcadas, con competencias muy dispares en equipamiento o personal. Esto es algo que los gobiernos locales no estaban preparados para asumir, con excepción de los gobiernos descentralizados de Quito, Guayaquil y Cuenca (Arroyo 2016, entrevista personal). Se puede colegir que el modelo de gestión descentralizada de este servicio no resultó ser, necesariamente, el más adecuado, pues las asimetrías de presupuesto y gobernanza son muy dispares.

La reducción de futuras vulnerabilidades se encuentra vinculada con el desarrollo de acciones tendientes a prevenir desastres posibles (Wright et al. 2020; McEntire et al. 2002; Herzog 2007). Este fenómeno fue particularmente agresivo con las edificaciones ya que más de 10.506 construcciones en áreas urbanas y 8157 en áreas rurales se vieron afectadas en diferente medida 
(EC SGR 2016). Por lo tanto, el sistema necesita incorporar protocolos de gestión de la infraestructura privada que incluyan acreditación de profesionales para actividades de inspección de viviendas que puedan determinar con rapidez si una infraestructura es habitable o no con el fin de acelerar el retorno a la normalidad de los habitantes de las zonas afectadas, así como facilitar el abastecimiento y el control del orden público.

\section{Conclusiones}

Las amenazas naturales, así como sus consecuencias, no se pueden anticipar ni controlar, y aunque el marco general de gestión de riesgos se ha caracterizado por enfrentarlos de forma reactiva (Fontana y Barberis Rami 2017), la gestión de emergencias debe incluir el manejo anticipado e integral de las redes de cooperación entre niveles de gobierno y con organizaciones de la sociedad civil, pues por sí mismo, un solo nivel de gobierno no puede atender todas las facetas de un evento catastrófico.

La investigación puso de relieve que, ante eventos como un terremoto de gran magnitud, los gobiernos presentan problemas para recuperar servicios básicos importantes, algo que sucedió en Ecuador, Perú o Chile en sus respectivos momentos. En el caso estudiado se puede sugerir que su capacidad de respuesta fue consecuencia de las acciones de fortalecimiento organizacional de varios sectores del Ejecutivo, como el eléctrico, entre otros, así como a la construcción de un sistema integral de gestión de riesgos y emergencias, mediante reformas institucionales, económicas y técnicas que, aunque positivo, no estuvo exento de falencias.

Este sistema debería realizar cambios en su diseño organizacional y en la forma de gestión operativa, para mejorar su integralidad, generando gobernanza plena y oportuna sobre la gestión del riesgo y emergencias para evitar los traslapes y vacíos identificados en la investigación.

A lo largo del análisis, se identificaron problemas de diseño organizacional, asignación de responsabilidades entre organizaciones públicas, diseño de protocolos y procedimientos, y mecanismos no adecuados de prevención y respuesta. 
Finalmente, los gobiernos locales son fundamentales para afrontar desastres relacionados con amenazas naturales y deben incrementarse los esfuerzos por fortalecer las capacidades que deben tener para enfrentar con mejores resultados los desafíos futuros. Se sugieren también mejoras en el diseño de protocolos y procedimientos, así como en los mecanismos de prevención y respuesta ante situaciones de emergencia o desastre.

\section{Apoyo}

Este trabajo es parte del proyecto de investigación: "Diseño organizativo del Ejecutivo y su impacto en la gestión pública" del Plan de Investigaciones del Vicerrectorado de Investigaciones de la Universidad del Azuay.

\section{Referencias}

Alto Comisionado de las Naciones Unidas para los Refugiados (ACNUR). 2017. "Terremoto en Ecuador. Un año después”. Accedido 11 de noviembre de 2020. https://bit.ly/3dHl QE3.

Báez Mancera, Luis Germán. 2013. "Mejoramiento de la gestión pública con ISO 9001:2008, estudio de caso". Scientia Et Technica 18 (1): 126-131. https://doi.org/10.22517/234472 14.8393 .

Banco Mundial. 1997. World Development Report 1997. Estudio institucional. Washington D. C.: Oxford University Press. https://bit.ly/31LFf9H.

Beydoun, Ghassan, Sergiu Dascalu, Dale Dominey-Howes y Andrew Sheehan. 2018. "Disaster Management and Information Systems: Insights to Emerging Challenges". Information Systems Frontiers 20: 14-28. 10.1007/s10796-018-9871-6.

Bowen, James D. 2020. "La construcción del Estado en Ecuador a través del ciclo económico: evaluando la "Revolución Ciudadana" durante el auge (y caída) de los recursos naturales". En Nuevos enfoques para el estudio de los Estados latinoamericanos, editado por Pablo Andrade, 169-189. Quito: UASB-E / Corporación Editora Nacional.

Cadillo, María Milagros. 2019. "El gobierno de las organizaciones que comparten recursos de uso común, retos y aproximación de los aportes del modelo antropológico". "In Defense of the Commons". The Seventeenth Biennial Conference. Lima. Accedido octubre de 2020. https://bit.ly/3nJFtzN. 


\section{Cristian Castillo Peñaherrera y Juan Santillán Berrones}

Castro Monge, Edgar. 2010. "El estudio de casos como metodología de investigación y su importancia en la dirección y administración de empresas". Revista Nacional de Administración 1 (2): 31-54. https://doi.org/10.22458/rna.v1i2.332.

Eburn, Michael. 2017. "Report. Australian Strategic Policy Institute". Accedido octubre de 2020. https://bit.ly/3mjupli.

EC. 2009. Decreto Ejecutivo 42. Registro Oficial 31, 22 de septiembre.

---. 2012. Decreto Ejecutivo 988. Registro Oficial 618, 13 de enero.

EC Ministerio de Economía y Finanzas (MINFIN). 2017. "Informe de ejecución presupuestaria. Ejercicio fiscal 2017. Informe anual”. Quito: MINFIN. Accedido octubre de 2020. https://bit.ly/2H2ymBw.

EC Secretaría de Gestión de Riesgos (SGR). 2014. Manual del Comité de Gestión de Riesgos. Accedido agosto de 2020. https://bit.ly/3jWL30b.

---. 2016. "Informe de situación No. 65 - Terremoto $7.8^{\circ}$ - Pedernales". Accedido agosto de 2020. https://bit.ly/3rVkb3w.

EC SGR e INSARAG. 2016. "Informe de los resultados del taller de lecciones aprendidas de las operaciones de búsqueda y rescate urbano. Montecristi, Manabí”. Accedido septiembre de 2020. https://bit.ly/3iWoGpx.

EC Secretaría Nacional de Planificación y Desarrollo (SENPLADES). 2016. Evaluación de los costos de reconstrucción. Sismo en Ecuador, abril 2016. Quito: SENPLADES. Accedido septiembre de 2020. https://bit.ly/2Nd0Dcf.

Ferrando, Francisco J. 2003. "En torno a los desastres naturales: tipología, conceptos y reflexiones". Revista INVI 18 (47): 15-31. Accedido septiembre de 2020. https://bit.ly/3j VE6LX.

Fontana, Silvia E., y Matías Barberis Rami. 2017. "Gestión del riesgo de desastres y sustentabilidad: aportes desde el enfoque de gobernanza". Revista Estado, Gobierno y Gestión Pública (29): 5-26. 10.5354/0717-8980.2018.50908.

Gellert-de Pinto, Gisela-Irene. 2012. "El cambio de paradigma: de la atención de desastres a la gestión del riesgo". Boletín Cientifico Sapiens Research 4: 13-17. https://bit.ly/2Fqsthb.

Henderson, Alexander, y Étienne Charbonneau. 2016. "An Examination of Emergency Services Research in Public Administration”. Public Administration Quarterly (SPAEF) 40 (3): 559-588. https://bit.ly/33CWSCq.

Herzog, Richard J. 2007. "A Model of Natural Disaster Administration: Naming and Framing Theory and Reality”. Administrative Theory \& Praxis 29 (4): 586-604. https://bit. ly/312nRW9.

Jung, Kyujin, Minsun Song y Hyung Jun Park. 2018. "The Dynamics of an Interorganizational Emergency Management Network: Interdependent and Independent Risk Hypotheses”. Public Administration Review 23: 225-235. 10.1111/puar.12993.

Kapucu, Naim. 2009. "Performance Under Stress: Managing Emergencies and Disasters". Public Performance \& Management Review 32 (3): 339-344. https://bit.ly/36GVP6a. 
---. 2011. "Developing Competency-Based Emergency Management Degree Programs in $\mathrm{Pu}-$ blic Affairs and Administration". Journal of Public Affairs Education 17 (4): 501-521. https://bit.ly/33xiFLE.

Lavell, Allan, y Chris Lavell. 2020. "El COVID-19: relaciones con el riesgo de desastres, su concepto y gestión". Red de Estudios Sociales en Prevención de Desastres en América Latina. Accedido enero de 2021. https://bit.ly/3r7H2IU.

Lein, Laura, Ronald Angel, Holly Bell y Julie Beausoleil. 2009. "The State and Civil Society Response to Disaster: The Challenge of Coordination". Organization \& Environment 22 (4): 448-457. https://bit.ly/2F70jI.

Levi-Faur, David. 2012. From Big Government to Big Governace? Oxford: University Press. 10.1093/oxfordhb/9780199560530.013.0001.

Lúquez de Camacho, Petra. 2016. "La teoría fundamentada: precisiones epistemológicas, teórico-conceptuales, metodológicas y aportes a las ciencias". Revista Cumbres 2 (1): 101114. https://bit.ly/3ofTJkf.

McEntire, David, Christofer Fuller, Chad Jhonston y Richard Weber. 2002. "A Comparison of Disaster Paradigms: The Search for a Holistic Policy Guide". Public Administration Review 62 (3): 267-281. https://bit.ly/2IgDO4q.

Mintzberg, Henry. 2003. Diseño de organizaciones eficientes. Buenos Aires: El Ateneo.

Moorthy, Ravichandran, Guido Benny y Sarjit S. Gill. 2018. "Disaster Communication in Managing Vulnerabilities". Journal of Communication 34 (2): 51-66. http://doi.org/10.17576/ JKMJC-2018-3402-04.

Nespeca, Vittorio, Tinna Comes, Kenny Meesters y Frances Brazier. 2020. "Towards Coordinated Self-Organization: An Actor-Centered Framework for the Design of Disaster Management Information Systems". International Journal of Disaster Risk Reduction 51: 18-32. 10.1016/j.ijdrr.2020.101887.

Oficina de Coordinación de Asuntos Humanitarios de las Naciones Unidas (OCHA). 2015. Guias de INSARAG, vol. I: Politica. Nueva York: ONU.

Ok Choi, Sang. 2004. "Emergency Management Growth in the State of Florida". State \& Local Government Review 36 (3): 212-226. https://bit.ly/3nJHY59.

Organización de las Naciones Unidas para la Alimentación y la Agricultura (FAO). 2008. "En tierra segura. Desastres naturales y tenencia de la tierra. El Ecuador: un país con elevada vulnerabilidad". Accedido agosto de 2020. https://bit.ly/3iUwQyI.

Organización Panamericana de la Salud (OPS/OMS). 2007. "Plan nacional de contingencia para enfrentar posible pandemia de influenza en el Ecuador". Accedido enero de 2021. https://bit.ly/3rVf978.

---. 2010. El terremoto y tsunami del 27 de febrero en Chile. Crónica y lecciones aprendidas. Santiago: AIRENA.

---. 2016. "Noticias e información para la comunidad internacional. Desastres. Preparación y mitigación en las Américas". Accedido octubre de 2020. https://bit.ly/3u5ozPa. 


\section{Cristian Castillo Peñaherrera y Juan Santillán Berrones}

Pazmiño, Andrea Cristina. 2020. "Análisis del Plan Nacional de Eficiencia Energética en el Ecuador". RIEMAT 5 (1): 28-34. https://doi.org/10.33936/riemat.v5il.2500.

Petak, William J. 1985. "Emergency Management: A Challenge for Public Administration". Emergency Management: A Challenge for Public Administration 45: 3-7. https://bit.ly/31 GBnqs.

Ramio, Carles. 1999. Teoría de la organización y administración pública. Madrid: Tecnos.

Rodríguez, Darío. 2002. Diagnóstico organizacional. Santiago: Ediciones Universidad Católica de Chile.

Rojas Hoppe, Carlos, y Silvia Díez Lorente. 2013. "El terremoto chileno del 27 de febrero de 2010: análisis preliminar de las consecuencias en la ciudad de Valdivia". Investigaciones Geográficas 7 (1): 139-153. https://doi.org/10.14198/INGEO2013.60.08.

Sanderson, David, y Ben Ramalingam. 2015. Nepal Earthquake Response: Lessons for Operational Agencies. Londres: ALNAP/ODI. https://bit.ly/3jX4AwH.

Schneider, Saundra. 2008. "Who's to Blame? (Mis) Perceptions of the Intergovernmental Response to Disasters". Publius 38 (4): 715-738. https://bit.ly/3jF3UvR.

UNESCO. 2011. "Manual de gestión del riesgo de desastre para comunicadores sociales. Una guía práctica para el comunicador social comprometido en informar y formar para salvar vidas". Accedido septiembre de 2020. https://bit.ly/3alVcR2.

Wallace, William, y Frank De Balogh. 1985. "Decision Support Systems for Disaster Management". Public Administration Review 45: 134-146. https://bit.ly/3759V1D.

Waugh, William L., y Gregory Streib. 2006. "Collaboration and Leadership for Effective Emergency Management". Public Administration Review 66: 131-140. https://bit. $1 \mathrm{y} / 2$ Yre76v.

Weichselgartner, Juergen. 2001. "Disaster Mitigation: The Concept of Vulnerability Revisited". Disaster Prevention and Management 10 (2): 85-94. 10.1108/09653560110388609.

Wright, Natalie, Lucy Fagan y Jostacio Lapitan. 2020. "Health Emergency and Disaster Risk Management: Five Years into Implementation of the Sendai Framework". Internacitional Journal of Disaster Risk 11: 206-217. https://doi.org/10.1007/s13753-020-00274-x.

\section{Entrevistas realizadas}

Arroyo, Éber. Tnte. Crnel., comandante Cuerpo de Bomberos de Quito, entrevista de la investigación, 11 de mayo de 2016.

Barriga, Patricio, secretario de Comunicación de la Presidencia, entrevista de la investigación, 7 de junio de 2016.

Dueñas, Susana, secretaria de Gestión de Riesgos, entrevista de la investigación, 7 de mayo de 2016.

Glas, Jorge, vicepresidente de la República, entrevista de la investigación, 7 de junio de 2016. Maldonado, Fernanda, viceministra de Inclusión Económica, entrevista de la investigación, 7 de junio de 2016. 
Navas, César, ministro Coordinador de Seguridad, entrevista de la investigación, 5 de julio de 2016.

Patiño, Ricardo, ministro de Defensa del Ecuador, entrevista de la investigación, 9 de mayo de 2016.

\section{Otras entrevistas que no estan citadas en el documento, pero que sirvieron como apoyo para realizar la presente investigación}

Albuja Obregón, Carlos, vicealmirante, comandante de Operaciones Navales y coordinador en territorio, entrevista de la investigación, 7 de julio de 2016.

Alcívar, Henry, funcionario Municipio de Pedernales, entrevista de la investigación, 19 de mayo de 2016.

Arteaga, Julio, funcionario Municipio de Pedernales, entrevista de la investigación, 19 de mayo de 2016.

Bravo Ochoa, Verónica, subsecretaria de Hábitat y Asentamientos Humanos (MIDUVI), entrevista de la investigación, 19 de junio de 2016.

Casanova Cedeño, Agustín, alcalde de Portoviejo, entrevista de la investigación, 19 de mayo de 2016.

Cedeño Rivadeneira, Fernando, exconsejero de Participación Ciudadana, entrevista de la investigación, 13 de junio de 2016.

Cucalón, Martín, Crnel., comandante Cuerpo de Bomberos de Guayaquil, entrevista de la investigación, 11 de mayo de 2016.

Morales Rosales, Karla, activista de derechos humanos, entrevista de la investigación, 19 de junio de 2016.

Pabón, Paola, secretaria nacional de Gestión Política, entrevista de la investigación, 20 de junio de 2016.

Paredes, Viviana, abogada y activista, entrevista de la investigación, 19 de junio de 2016.

Ramírez, Oswaldo, Crnel., primer jefe del Cuerpo de Bomberos de Cuenca, entrevista de la investigación, 11 de mayo de 2016.

Ricaurte, Peggy, Subsecretaría Protección Social Especial (MIES), entrevista de la investigación, 23 de junio de 2016.

Salcedo Faytong, Carolina, directora provincial del Guayas (MIDUVI), entrevista de la investigación, 19 de junio de 2016.

Serrano, José, ministro del Interior, entrevista de la investigación, 23 de junio de 2016.

Toalá, Marcelo, funcionario Municipio de Pedernales, entrevista de la investigación, 19 de mayo de 2016.

Zambrano Cedeño, Jorge, alcalde de Manta, entrevista de la investigación, 19 de mayo de 2016.

Zambrano Intriago, Fernando, coordinador Zonal 4 (MIDUVI), entrevista de la investigación, 19 de junio de 2016. 